\title{
The Contrast of Chinese and English in the
}

\section{Translation of Chinese Poetry}

\author{
Ning Li \\ Continuing Education College \\ Beijing Information Science \& Technology University \\ Beijing 100076, China \\ E-mail: emmalining@126.com
}

\begin{abstract}
Chinese poetry is the soul of Chinese literature and Chinese culture. A good translation of a Chinese verse can promote the prevalence of Chinese culture. In the translation of Chinese poetry, translators should not only keep the characteristics of Chinese poems, but also embody the English characteristics. This article analyzed some versions of translation and proposed factors affecting the translation of Chinese poetry.
\end{abstract}

Keywords: Chinese Poetry, Translation, Contrast

\section{Introduction}

Chinese poetry is one of the most important parts in Chinese literature. Since The Book of Odes, the first poetry collection written by Confucius in the spring-autumn period was known by people, Chinese poetry has developed quickly. In the Tang Dynasty, Chinese poetry was at its peak. In this period, many immortal poems were written and had great influence on the later Dynasties. In the later Dynasties, Chinese poetry went on its development. But because of the development of other literary styles - Yuan Songs (drama created mainly in Yuan Dynasty), novels, and essays, poetry is no longer the major literary style. In this article, I'd like to talk about the contrast of Chinese and English in the translation of Tang-poems. Tang-poems has very strict phonological format. In each stanza, there are five (in quatrains) or seven (in regulated verses) words. Using limited words to express unlimited sense is the major characteristic of Chinese poetry. Now, seldom people have the interest and capability to write Chinese poetry. But man still likes it very much. Even those young children are proud of reciting Chinese poetry. At the same time, as the soul of Chinese literature, Chinese poetry is also loved by foreign Chinese learners and those who are interested in Chinese culture. It has been translated into English by both Chinese and foreigners since long before. However, Chinese is very different from English in many aspects. Chinese is an implicit language, which takes the parataxis and the rhythm as the most important thing while English is quite explicit. Parataxis means instead of using morphological connections, people use functional words or logical relationship of each component to compose a sentence (Pan, 1997: pp. 334). Conforming to syntactic rules is what English concern. In the form of language, Chinese is more flexible while English is very rigid. (Pan, 1997). These differences determine that a good translation should keep the implicit of Chinese poetry on the basis of not ruining the English rules. Some translators have succeeded in connecting the different peculiarities of the two languages but in most cases, it is a real task. In this article, I'd like to state how to harmonize the contrast of Chinese and English in the translation of Chinese poetry.

\section{To keep the Chinese poetry's characteristics}

I have mentioned the characteristics of Chinese poetry in the previous paragraph. The rhythm and parataxis is what Chinese concern. English is a language with syntax. The English version should keep these syntactical rules. How to keep rhythm and parataxis on the basis of sticking to the syntax of English?

\subsection{The corresponding rhythm}

Chinese poetry has a very strict rhythm. In each stanza, the number of words is fixed, and the rhyme should be homologous in the even stanzas. When we translate it into English, whether we should try to keep the Chinese rhythm or conform to English syntax is a major concern of translators. Some of them managed to keep the Chinese rhythm. For example:

Ji Sheng Mao Dian Yue, Ren Ji Ban Qiao Shuang

In this poem, no verb is used. But we Chinese haven’t any difficulty in understanding it as "At a frosted night, the cock 
is crowing outside the thatch inn and there are seldom people on the wood bridge." However, someone has ever translated it as,

Cock crow thatch inn moon.

Man trace wood bridge frost. (Zhang, 1993, pp. 19)

Readers could possibly find this translation is of fun, but will English native speakers understand it?

Other translators give priority to English syntax in their translations. For example,

Deng Guan Que Lou (Wang Zhi Huan)

Bai Ri Yi Shan Jin, Huang He Ru Hai Liu.

Yu Qiong Qian Li Mu, Geng Shang Yi Ceng Lou.

$\mathrm{Xu}$ (1990, pp. 31-32) translated it as

An Ascent to Stork Hall

The setting sun behind the mountains glows

The muddy Yellow River seawards flows.

If more distant views are what you desires.

You simply climb up a storey higher.

It's a typical English poem with a rhyme of aabb. Though it's not completely identical to the original format, both the form and the expression are all very successful. It can be called a work of art. In another example, Zhu (1995) translated "Quan Fei Shui Sheng Zhong, Tao Hua Dai Yu Nong" as,

A dog,

A dog barking,

And the sound of the rushing water.

How dark and rick the peach flowers after the rain.

The author used some present participle instead of verbs making the English version very vivid with the barking of the dog and the rushing of the water

But sometimes it's really very difficult to make a compromise between rhythm of Chinese poem and English syntax rules. In such occasions, the obedience to the implicit sense of the original poem is very important. For example,

Xun Yin Zhe Bu Yu (Jia Dao)

Song Xia Wen Tong Zi, Yan Shi Cai Yao Qu.

Zhi Zai Ci Shan Zhong, Yun Shen Bu Zhi Chu.

W. Bynner (1995) translated it as

When I question your pupil, under a pine tree.

"My teacher," he answered, "went for herbs"

"But toward which corner of the mountain,

How can I tell through all these clouds"? (cf. Zhang, 1994)

In this translation, we can't find a slightest trace of the original poem. Bynner translated "Yan Shi Cai Yao Qu, Zhi Zai Ci Shan Zhong, Yun Shen Bu Zhi Chu" as a direct answer of the little boy. However, it's still a good version for the readers can both understand the implicit meaning of the poem and enjoy the mood of the poet.

Rhythm is very important in Chinese and Chinese poetry. But when Chinese poetry is translated into English, it doesn't matter whether we keep the rhythm or not. The grasping of the implicit sense is more important than just keeping the form.

\subsection{The realization of parataxis}

In Chinese poetry, the realization of parataxis is very important. Poets prefer using logic relationship to grammar rules in composing stanzas. In order to be as compact as possible, poets may use some incomplete sentences. The readers could complement these incomplete parts according to their imagination. However, English is an explicit language, the supplement of the omitted part in the original poem seems necessary. Then is there a standard for good translations? In my opinion, a good translation should be coincide on logical relationship with the original poems. In other words, translators should attach importance to some key meaning clusters of the Chinese poems in narrow sense; while in broad sense, they should give superiority to the implicit sense and the mood of the poets. 
2.2.1 Focusing on key meaning clusters of a Chinese poem

Let's make an comparison of the following translations.

Yue Luo Wu Ti Shuang Man Tian,

Jiang Feng Yu Huo Dui Chou Mian. (Zhang Ji )

In Bynner's Translation,

While I watch the moon go down, a

Crow caws through the forest,

Under the shadows of maple trees, a

Fisherman moves with his torch.

----Translated by Bynner ( $c f$. Zhang, 1994, PP. 22)

Zhang Ting-Chen and Wilson translated as,

The setting moon, a cawing crow, the

Frost filled sky

River-maples, fisherman flares, and troubled sleep

---- Zhang Ting-Chen and Wilson (cf. Zhang and Wilson, 1991: 10-11)

In Xu Yuan-Zhong's translation,

The moon goes down the cows caw in

The frosty sky

Dimly-lit fishing boats' neath maples sadly lie

----Xu Yuan-Zhong (cf. Zhang, 1994: 22)

The key meaning cluster in this poem, is "Dui Chou Mian", for it can reflect the mood of the poet the best. In Bynners' version, "Dui Chou Mian"has been omitted, so the two stanzas have become a description of the scenery. Zhang and Wilson's (1991) version adopted the rhythm of the original poem, using six nouns in their version. We Chinese native speakers may understand it, but whether native English speakers can understand it is a big problem. This version is not coincident with English syntax. The ellipsis of the subject may embarrass the readers. Besides, "Dui" 'hasn't been translated. While the third version seems the best one in all the translations. Xu used "personification" to endow nature with the quality of human beings, which pull the traveler in the boat and the scenery out the boat together. The third version can really help readers taste the poet's loneness when he lay sadly at night. So the translation of the key meaning clusters can help readers make a better understanding of the whole poem.

2.2.2 Focusing on implicit sense and poets' mood

In the translation of Chinese poetry, grasping of implicit sense is the most important thing. The logical relationship of each component of the poem is the soul of a good translation. Though grammar rules have often been considered in English, the coincidence in implicit sense cannot be ignored. Differences in understanding the implicit sense may emerge different effect.

Xun Yin Zhe Bu Yu (Jia Dao)

Song Xia Wen Tong Zi, Yan Shi Cai Yao Qu.

Zhi Zai Ci Shan Zhong, Yun Shen Bu Zhi Chu.

Bynner translated it as,

A Note Left for an Ascent Recluse

When I question your pupil, under a pine tree.

"My teacher," he answered, "went for herbs"

"But toward which corner of the mountain,

How can I tell through all these clouds"? ---- Bynner (cf. Zhang 1994, pp. 25)

I met your boy' neath a pine tree.

"My master's gone for herbs," says he 
"Amid the hills I know not where,

For clouds have veiled them here and there."

---Xv Yuan-Zhong (cf. Zhang 1994, pp. 25)

I questioned a boy underneath a pine tree,

Thus answered he "The master is gone to gather herbs,

Must be right here in the mountain.

The clouds are thick; I can't tell where."

----Lu Zhi-Wei (cf. Zhang 1994, pp. 25)

Poets use limited words to express unlimited sense. The translators should try to supplement those words which are unsaid by the poets through their imagination. This poem is a very famous poem in Chinese literature. It expresses the change of visitor's state of mind and the quality and style of the visited. All the three versions can describe these two aspects well. But the third version is the best. After reading the version, we seem to see a little child is answering a visitor frolicly. In the third sentence " must be right here in this mountain" gives the visitor a gleam of light, but in the last sentence "the clouds are thick; I can't tell where" the gleam of light become desperation. The change of visitor's state of mind seems experienced by our readers.

So, in the translation of Chinese poetry, a completely corresponding rhythm is not necessary, but parataxis is very important. The English version should attach importance to the key meaning clusters and grasp the implicit meaning of the original poem. The logical relationship in Chinese should be full reflected in the English version.

\section{To embody the English characteristics}

English is a kind of syntax language, which determines that the English version of Chinese poetry should and must have the characteristics of morphological language. At the same time, English is a kind of Subject-Predicate language (Pan, 1997), so an English sentence should have both the subject and the predicate. The subject and the predicate should be identified. Subjects have singular form and plural form. The predicate parts may change according to the tense. Chinese haven't morphology, so it doesn't need tenses, singular or plural form, and the identification of the subject and the predicate. Chinese is a kind of Topic - Comment language (Pan, 1997). Each part of speech can be the topic, so both the subject and the object can be omitted. On the other hand, English is very rigid, so the morphological rules must be submitted, while Chinese is flexible, and logical relationship is most important. Because of the fixed format of Chinese poetry, many components have been omitted, but it won't have an influence on the understanding of the Chinese poetry. But when we translated it into English, only by complementing the omitted grammar components, the English version can be well expressed, so we should try to make a complementary of these grammar components.

\subsection{The supplement of subject}

The ellipsis of subject is often found in Chinese. In Chinese poetry, we make an effort to use fewer words to express deep meaning. So the ellipsis of subject is very familiar to Chinese speakers. However, subject is a very important component in English for English is a kind of Subject-Predicate language. So choosing a good subject is very important in translating Chinese poetry. Look at this example,

Xin Jia Niang (Wang Jian)

San Ri Ru Chu Xia, Xi Shou Zuo Geng Tang.

Wei Yin Gu Shi Xing, Xian Qian Xiao Gu Chang.

In Chinese, we will never take the executor of the action seriously. What we know is a newly married woman tries to serve for her mother-in-law. But if we must give it a subject, who is it? W.J Fletcher (1926) translated it as,

Now married three days, to the kitchen I go,

And washing my hands, a fine broth I prepare.

But what kind of taste auntie likes, I don't know,

So deed to my sister-in-law, the first share.

In this version, Fletcher used "I" as the subject. In his version, a traditional Chinese woman is telling us her daily life after marriage. Xu Zhong-Jie translated it as,

Three days after the wedding,

Her duties as bride, she saw.

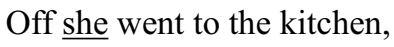


Made soup for mother-in-law.

Not knowing what's to her taste,

What's the best to do, she saw.

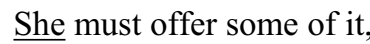

For trial to sister-in-law. (Xu 1990, pp. 31-32)

In this version, the author tells us a story of a traditional Chinese woman's marriage life in the third-person. The different personal pronoun brings us different feelings. We may say the first translation is more vivid, but we can't say the second is a bad one.

But in another case, translator made mistakes in deciding subjects. For example,

Xiao Yue Guo Can Lei, Fan Xing Su Gu Guan.

Bynner translated it as,

The moon goes down behind a ruined fort,

Leaving star-clusters above a gate. (cf Zhu, 1995, pp. 41)

In this version, we can see that the author didn't know what should be the real subject in this poem. In his opinion, "the moon" and "the star" are the subjects. In fact, the real subject should be the person who is passing by the ruined fort. The sense of it is a man passed by a ruined fort in the early evening. In Zhang Ruogu's version,

By waning moon, you'll cross the desolate battle-ground,

Underneath myriad stars, you'll sleep at the old gate,

And sleep at the old gate underneath the clustered star. (Zhu, 1995, pp. 41)

The translator has really understood the original poem.

\subsection{The supplement of predicate.}

Predicate is the quintessence of English sentences. It can't be absent almost in any condition. But in Chinese, predict is not always needed. Some nouns in Chinese can express a dynamic sense. So choosing an accurate verb to be the predicate will make the translation more vivid.

Chang'an Yi Pian Yue, Wan Hu Dao Yi Sheng. (Du Fu)

In this poem, we can see "Chang'an "is a noun, "Yi Pian" is a number-plus-classifier expression. "Yue" is another noun. In the second sentence, "Wan Hu" is a number-plus-classifier expression. "Dao Yi Sheng" is a noun too. In these two stanzas, no verb was used. Let's look at the English versions,

In Bynner's version,

A slip of the moon hangs over the capital,

Ten thousand washing-mallets are pounding. ( $c f$ Zhu, 1995, pp. 40)

Yang Yixian and Dai Naidie's version is,

The moon shires upon Chang'an city

From all houses holds the sound of pounding clothes. (cf. Zhu. 1995, pp. 40)

$\mathrm{Xu}$ Zhong-Jie's version is,

Over Chang'an hangs the moon

Callous and cold

For each house hold thumping sounds issue forth. (cf. Zhu, 1995, pp. 40)

Now let's make a compare of the three versions. In the first sentence, both Bynner and Xu Zhong-jie use "hangs over", while Yang \& Dai deal it with "shires upon". The verbs used in the three versions share the same meaning. We don't discuss it in detail, here. We shall pay more attention to the verbs in the second sentence. The Yang \& Dai used "holds", while Xu used "hold...issue forth". "issue forth" has the meaning of "Fa Chu". It's a dynamic verb, so it's better than the other one. However, I prefer Bynner's version. He adopted a present progressive " are pounding" as the predicate. Readers seem to have heard the pounding of the washing-mallets after reading his version. They may draw a beautiful picture on their minds that in a peaceful night, the moon hangs over. The pounding of the washing mallets can be heard. Many families are enjoying their pacific and beautiful life. "hangs over" is a static verb phrase, while " are pounding" is dynamic. The connection of static state and the dynamic one makes the translation very colorful. 


\subsection{The identification of the subjects and predicates}

In English, we should distinguish the singular form from the plural form in subjects. The form of predicates should be identified with the subjects. But Chinese is a Topic-Comment language. There is only logic relationship between the topic and the comment. In our translation, we must try to keep the identification of the subjects and predicates. See the example,

Bai Tou Gong Nu Zai, Xian Zuo Shuo Xian Zong.

H.A Giles translated it as,

One white-haired dame

An emperor's flam

Sits down and tells of bygone hours. (cf. Zhu, 1995)

In this version, we may consider it as a deserted woman who has been the emperor's dame is telling the poet the story about the emperor.

W.J.B Fletcher's translation,

Only some withered dames with whitened hair remain,

Who sit there talking of mystic monarch dead. (cf, Zhu, 1995, pp. 39)

It tells us many old ladies who have been the emperor's dames sit together idly and talk about the mystic monarchy dead. We Chinese won't think too much about how many dames there are, but in English version, the number of the dame cannot be ignored. One dame, or several dames can bring different feelings, but it's very hard for us to tell which version is better.

\subsection{The tense of the predicate}

Chinese verse won't be limited by a specific time or space. It will leave the readers more space to associate with. Tense is indispensable in English. English speakers would like to use tense to express accurate time. Something happened in the past should be expressed in past tense, while things happens now should be expressed in present tense. Except tense, mood, aspect and voice is often be used too. In our translation, we must consider when does the event in the poem happen.

Yue Zhong Lan Gu

Yue Wang Gou Jian Po Wu Gui, Yi Shi Huan Jia Jin Jin Yi.

Gong Nu Ru Hua Man Chun Dian, Zhi Jin Wei You Zhe Hu Fei.

The first three stanzas happened in ancient time. The last one returns to the present scenery.

Yuan-Chong's version,

The king of Yue returned, having destroyed the foe.

His loyal men came home, with silken dress aglow.

His palace thronged with flower-like ladies fair.

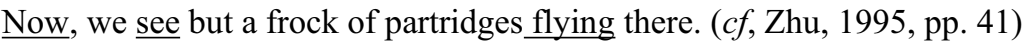

In the first three stanzas, the translator adopts the past tense "returned, having destroyed, came thronged" to recall the things happened long before. In the last stanza, a "now" and "see...doing" brings us to present.

Sometimes, the translators used different tense to describe the same scene.

Chun Xiao (Meng Hao Ran)

Chun Mian Bu Jue Xiao, Chu Chu Wen Ti Niao,

Ye Lai Feng Yu Sheng, Hua Luo Zhi Duo Shao.

Zhang Ting-Chen and Bruce $\mathrm{M}$ Wilson translated it as,

Oversleeping in spring I missed the dawn.

Now everywhere the cries of birds are heard.

Tumult of wind and rain had filled the night.

How many blossoms fell during the storm? (Zhang and Wilson, 1991, pp. 22-23)

$\mathrm{Xu}$ Zhong-jie translated it as,

One slumbered late in the morning in spring. 
Everywhere, one hears birds' warble or sing.

As the night advance, rain spatters, winds moan.

How many flowers have dropped? Can that be known? (XU, 1990. pp. 28)

In the first stanza, both Zhang\&Wilson and Xu used past tense. Present tense was used by both of them in the second stanza, indicating that the bird is singing now. However, In the third stanza, Zhang \& Wilson used past perfect tense to show there was a strong storm last night, and the last stanza, a present tense used to tell what the author thinks now. Xu's the third and the fourth stanza can be taken as an adverbial clause. Present tense in the subordinate clause describes a fact and the present perfect used in the main clause indicates that the rain spattered, winds moaned in the past. The tenses the translators selected are quite different, but their understanding about the original poem is quite the same.

\subsection{The use of functional words}

Both Chinese speakers and English speakers like using functional words in their sentences. But functional words in the two languages have differences in essence. The functional words in English are the complement of morphology, while in Chinese, it's the product of semantic vacancy and the complementary of rhythm. (Pan, 1997, pp. 304) The functional words in English are rigid, and can't be omitted or replaced. But functional words in Chinese are very flexible. It can be filled or omitted randomly. The characteristic of Chinese verse is to use few words expressing deep meaning. So functional words are often omitted. When we translate them into English, we should supplement the functional words. Look at this poem,

Ji Sheng Mao Dian Yue, Ren Ji Ban Qiao Shuang (Wen Ting Jun)

Six nouns are used here, someone translated it as,

Cock crow thatch inn moon.

Man trace wood bridge frost.

It's not typical English sentences. It's an interesting attempt, but in most cases, we won't translate them like this. In our translation, we will supplement the omitted components to show the logic relationship of original poem. Functional words here are very necessary.

Behind the thatched inn, crewing cock and setting moon.

Upon the planked bridge, footprints in the frost. (Zhu, 1995, pp. 41)

The three functional words can help readers to understand the relationship of things. Behind the thatch inn, the cock is crowing. The moon has been setting, and the footprints step upon the planked bridge in the frost weather. Let me give you another example,

Jue Ju (Du Fu)

Liang Ge Huang Li Ming Cui Liu, Yi Hang Bai Lu Shang Qing Tian.

Chuang Han Xi Ling Qian Qiu Xue, Men Bo Dong Wu Wan Li Chuan.

Dou Hua-Zhan's version is,

Two gold orioles chirp on the emerald willow, outside the sill.

And a covey of white egrets fly towards the azure sky.

From the windows one sees eternal snow on the western hill.

At the door a boat bound for the east is tied nearby. (Dou, 1992, pp. 44)

There is no any functional word in the original poem, but there are seventeen functional words in the version. So the use of functional words is one of the major differences between Chinese and English.

\section{Conclusion}

The contrast of Chinese and English can help us to know the two languages better. It not only can help us to learn or teach English as a foreign language, but also can help us a lot in translation. Chinese verse is the soul of Chinese literature and Chinese culture. It's one of the most important parts in culture communication. A good translation of a Chinese verse can promote the prevalence of Chinese culture. What are the most important principles in our translation? The problem is always disputed in the field of linguistics and translation. It's very hard to connect the Chinese characteristics with the English ones, for the two languages are so different. Some people think that keeping the characteristics of Chinese is the most important thing, so they translate poems with Chinese rhythm. But other people believe that the versions should be typical English sentences, or it will be helpless for English speakers to understand it. In my opinion, the versions should be helpful for readers to understand the sense of the original poems. The sense will 
be taken as the first important thing. In form, the versions are required to be coincident with the English rules. It should have the change of person, number, mood, tense, aspect and voice. Functional words should be supplemented according to the English rules. Keeping the Chinese rhythm is good, but not very necessary.

\section{References}

Dou, Huazhan. (1992). Chinese poems in English verse. Foreign Language, 1.

Fletcher, W. J. (1926). Gems from Chinese verse. Shanghai: Shanghai Commercial Press.

Herbert A Giles. (1901). A History of Chinese Literature. London: William Heinemann.

Liu, Chongde. (1999). The Characteristics of Huang Bofei's English verse of Chinese poems. Foreign Language, 3.

Lv, Shuxiang. (1980). Notes for Chinese poems in English Verse (in Chinese). Shanghai: Shanghai Foreign Language Education Press.

Pan, Wenguo. (1997). Essentials of the Contrast of English and Chinese (in Chinese). Beijing: Beijing Language and Culture University Press.

Sun, Zhu (ed.) and Jin, Xingyao (annotate). (1980). New Annotation for 300 Chinese Tang Poems (in Chinese). Shanghai: Shanghai Ancient Books Publishing House.

Xu, Zhongjie. (1990). 200 Chinese Tang Poems in English Verse (in Chinese). Beijing: Beijing Language and Culture University Press.

Zhang, Baohong. (1994). Significance Recognition of Chinese poems in English verse. Foreign Language, 2.

Zhang, Shaoxiong. (1993). Review on the emergence of Chinese poems in English Version (in Chinese). Foreign Language, 3.

Zhang, Tingchen and Wilson. (1991). 100 Chinese Tang Poems (in Chinese). Beijing: The Commercial Press.

Zhu, Hui. (1995). Grammar issues of Chinese poems in English version (in Chinese). Foreign Language, 6. 